\title{
The role of pembrolizumab in the treatment of advanced non-small cell lung cancer
}

\author{
Giuseppe Santabarbara ${ }^{1}$, Paolo Maione ${ }^{1}$, Antonio Rossi ${ }^{1}$, Giovanni Palazzolo ${ }^{2}$, Cesare Gridelli ${ }^{1}$ \\ ${ }^{1}$ Division of Medical Oncology, “S. G. Moscati” Hospital, Contrada Amoretta 83100, Avellino, Italy; ${ }^{2}$ Division of Medical Oncology, "ULSS 15 \\ Cittadella”, via Casa di ricovero, 4035013 Cittadella, Padova, Italy \\ Contributions: (I) Conception and design: All authors; (II) Administrative support: All authors; (III) Provision of study materials or patients: All \\ authors; (IV) Collection and assembly of data: All authors; (V) Data analysis and interpretation: All authors; (VI) Manuscript writing: All authors; (VII) \\ Final approval of manuscript: All authors. \\ Correspondence to: Cesare Gridelli. Division of Medical Oncology, “S. G. Moscati” Hospital, Contrada Amoretta 83100, Avellino, Italy. \\ Email: cgridelli@libero.it.
}

\begin{abstract}
Lung cancer is the leading cause of death cancer related worldwide. The standard therapies have unmet medical needs both due to the limited activity and relevant toxicity of platinum-based chemotherapy and to the low frequency of specific alterations required to use targeted therapies. Immune checkpoint inhibition due to restoring the immune system's capacity to eradicate tumors is undergoing in extensive investigation in non-small cell lung cancer (NSCLC) as a new treatment approach. Programmed cell death protein-1 (PD-1) and its ligand, programmed cell death-ligand 1 (PD-L1) have recently led to significantly and durable improvements in the clinical outcome of several kind of tumors including lung cancer. Pembrolizumab, approved by the U.S. FDA for the treatment of advanced NSCLC progressed after other therapies and with expression of PD-L1, has demonstrated durable response and prolonged overall survival (OS) especially in patients with high PD-L1 expression. Further investigation are needed to improve treatment outcomes through combination of immunotherapy or combined with other targeted therapies.
\end{abstract}

Keywords: Non-small cell lung cancer (NSCLC); programmed cell death protein-1 (PD-1); programmed cell death-ligand 1 (PD-L1); pembrolizumab

Submitted Apr 20, 2016. Accepted for publication May 02, 2016.

doi: $10.21037 /$ atm.2016.05.64

View this article at: http://dx.doi.org/10.21037/atm.2016.05.64

Lung cancer accounted for an estimated $13 \%$ of total cancer diagnoses and is the most relevant cause of death cancerrelated worldwide. Non-small cell lung cancer (NSCLC) reaches about the $85 \%$ of lung cancer cases, with a prevalence of non-squamous histology (1). Platinum-based chemotherapy remains the standard treatment for most patients with 1-year survival probability of about $40 \%$ with no patients alive at 5 years. This kind of therapy is indicated for patients with good performance status (ECOG 0-1) due to the induction of relevant toxicity (i.e., renal, neurological and hematologic toxicities) who limit its use in patients with poor performance status and comorbidities (2).

The discovery of driver mutations in genomic subset of patients such as epidermal growth factor receptor (EGFR) activating mutations or anaplastic lymphoma kinase (ALK) translocations has led to development of molecular targeted agents able to improve clinical outcomes in this subset of patients. Indeed the EGFR tyrosine kinase inhibitors (TKIs) such as erlotinib (3), gefitinib (4), afatinib (5) and, similarly, ALK inhibitor crizotinib (6) have demonstrated marked superiority over chemotherapy with a median survival approaching 2 years. Many unmet medical needs remain because these specific alterations are estimated in about $10-15 \%$ of NSCLC patients in western population for EGFR mutations and in 2-4\% for ALK translocations. In adding is demonstrated a "second-site mutation" in exon 20, T790M, in about $50 \%$ of EGFR-mutated lung cancers treated with erlotinib or gefitinib or afatinib. It confers an acquired resistance to these drugs but a potential sensitivity to a third generation of anti EGFR-TKIs (7). 
The immune system plays a role in control cancer identifying and destroying foreign or abnormal cells $(8,9)$. This mechanism of immunosurveillance involves both innate and adaptive immune system including natural killer (NK) cells, CD4+ T-cells, CD8+ T-cells and macrophages. T-cell activity and regulation are critical to tumor development because CD8+ T-cells are capable to eliminate tumor cells. In several studies this kind of T-cells needed elimination of regulatory T-cells, support of interleukin-2 or other immune-stimulating cytokines to maximize the elimination of tumor cells (10-12). Thus, although T-cells have the ability to eradicate tumors, one hallmark of cancer cells is immune evasion, in which the system does not mount an effective antitumor response. This is due to the creation by tumor cells of immunosuppressive environment enabling them to grow or spread unchecked. There are many evidences that cancer cells including lung cancer are able to evade immune control through many mechanisms such as recruitment of inhibitory immune cells (e.g., CD4+ regulatory T-cells), antigen presentation defects, secretion of immunosuppressive cytokines in the tumor microenvironment (e.g., interleukin-10, transforming growth factor- $\alpha$ ) and cellular immune dysfunction through modulation of immune checkpoint pathways (9,13-16).

Regulatory T-cells, immunosuppressive cytokines and immune checkpoints, including the cytotoxic T-lymphocyte antigen 4 (CTLA-4), programmed cell death protein-1 (PD-1) and its two ligands [programmed cell death-ligand 1 (PD-L1) and PD-L2] are the main inhibitory forces (17).

Immune checkpoints are receptors localized on cell surface that, when bound their ligand, modulate immune response. In healthy, the immune checkpoint molecules negatively regulate the immune system to maintain peripheral self-tolerance and prevent autoimmunity $(14,18)$. The CTLA- 4 and PD-1 are the best-characterized immune checkpoint pathways; they suppress T-cell activity in different ways: CTLA- 4 regulates T-cell activity at an early stage whereas PD-1 regulates later effector T-cell activity within tissue and tumors (19). CTLA-4 is a receptor expressed on activated T-cells, its interaction with ligands B7-1 (CD80) and B7-2 (CD86) on antigen-presenting cells (APC) leads to a downregulation of T-cells activation (20-22). The interaction between PD-1 receptor expressed also on activated T-cells and its ligands, PD-L1 (also called B7-H1) and PD-L2 (also called B7-DC) downregulates T-cells activity to prevent tissue damage at the sites of chronic inflammation and unwanted or excessive immune response, including autoimmune reactions (23). PD-L1 is expressed at low levels on various non-hematopoietic tissues, most notably on vascular endothelium, whereas PD-L2 is expressed only on APCs found in lymphoid tissue or chronic inflammatory environments. Tumor cells by upregulation of the PD-L1 and PD-L2 expression can evade the immune activation and subsequent recognition and destruction by immune system. High expression of PD-L1 on tumor cells has been found to correlate with poor prognosis and survival in various cancer types, including renal cells (24), pancreatic (25), hepatocellular (26) and ovarian carcinoma (27). PD-1 is also induced on other activated non-T-lymphocytes subsets, including NK cells and $\mathrm{B}$ cells, which may be inhibited by tumors expressing PD-L1 or PD-L2. Studies demonstrated that PD-L1 binds CD80 besides PD-1, and PD-L1/CD80 interaction also delivers inhibitory signals in T-cells (28).

To date, the PD-L2 expression on the surface of cancer cells seems to have a not relevant clinical impact (12) whereas the expression of PD-L1 promotes, due interaction with PD-1, apoptotic death of activated tumor antigen-specific human T-cells in vitro and antigen-specific T-cells in a mouse tumor model (29). Many studies have associated PD-L1 expression on tumor cell surface with larger tumor size or as a risk factor for poorer outcomes in terms of progressionfree survival and overall survival (OS) (30). The inhibition of the interaction between PD-L1 and PD-1, by the use of specific monoclonal antibodies against PD-1 or PD-L1, has been able to enhance local T-cell response restoring the therapeutic immunity function and leading to regression of tumor growth in preclinical animal models $(31,32)$. In the clinical setting, the use of antibodies able to block PD-1/ PD-L1 interaction have been reported to produce objective response rates up to $38 \%$ in patients with metastatic solid tumors, about $20 \%$ in NSCLC, with tolerable safety profiles $(33,34)$. Although NSCLC is not considered a typical immune-responsive tumor, squamous histology is associated with high frequency of mutation and formation of multiple novel tumor antigens, which may make it more sensitive to immune recognition $(35,36)$. In this kind of tumor have been identified some inactivating mutations in the locus human leukocyte antigen-A (HLA-A) of major histocompatibility complex (MHC) class 1 gene that could provide a way to avoid the recognition/elimination by immune system (37).

Several antibodies against PD1 and PD-L1 have shown their antineoplastic activity in NSCLC. Nivolumab, a fully human IgG4 PD-1 immune-checkpoint-inhibitor antibody, in a phase 1 expansion cohort of patients with pretreated NSCLC reached an overall response rate (ORR) of $17 \%$ 
with 9.9 months median OS without difference in squamous and non-squamous histology (38). These results were confirmed in subsequent phase 2 and $3(39,40)$ trials granting the approval of nivolumab for the treatment of patients with squamous and non-squamous metastatic NSCLC after failure of platinum-based chemotherapy worldwide.

Pembrolizumab is a humanized IgG4 monoclonal antibody that prevents the PD-1 to bind PD-L1 and PD-L2 thus preventing the formation of inhibitory signals in T-cells with restoring of tumor recognition by $\mathrm{T}$-cells CD8+. The first-in-human experience conducted by Patnaik et al. in a phase 1 trial has showed that pembrolizumab can be administered safely at doses of 1 to $10 \mathrm{mg} / \mathrm{kg}$ every 2 weeks and demonstrated evidence of antitumor activity in several types of tumors including Merkel cell carcinoma (MCC), melanoma and NSCLC (41). In September 2014 pembrolizumab, based on data from 173 participants in a multicenter, open-label, randomized (1:1), dose-comparative, activity-estimating cohort of trial 1 , was approved by FDA for the treatment of unresectable or metastatic melanoma and disease progression following ipilimumab and a BRAF inhibitor in patients who were BRAF V600 mutation-positive. The ORR was 24\% in the $2 \mathrm{mg} / \mathrm{kg}$ arm, consisting of 1 complete response and 20 partial responses. Among the 21 patients with an objective response 18 (86\%) had ongoing responses with durations ranging from $1.4+$ to $8.5+$ months, which included 8 patients with ongoing responses of 6 months or longer. There were objective responses in patients with and without BRAF V600 mutation-positive melanoma. Similar ORR results were observed in the $10 \mathrm{mg} / \mathrm{kg}$ arm (42). In October 2015 based on data from KEYNOTE-001 trial (43) the U.S. Food and Drug Administration (FDA) has granted accelerated approval for pembrolizumab to treat patients with metastatic NSCLC whose disease has progressed after other treatments and with tumors that express a protein called PD-L1.

\section{Results from clinical trials}

\section{KEYNOTE-001 (43)}

In this trial 495 patients with locally advanced or metastatic NSCLC were randomized to receive pembrolizumab at the dose of either 2 (6 patients) or $10 \mathrm{mg} / \mathrm{kg}$ (287 patients) every 3 weeks or $10 \mathrm{mg} / \mathrm{kg}$ (202 patients) every 2 weeks. About $80 \%$ of patient had non squamous histology and had received more than two precedent lines of chemotherapy. In this trial ORR was $18 \%$ for patients pretreated and $24.8 \%$ ( $n=101$ patients) for treatment-naïve patients, $21.8 \%$ of all patients had a stable disease as the best overall response. Patients with squamous histology (85 patients) showed an ORR of $23.5 \%$ while for patients with non-squamous histology the ORR was $18.7 \%$. Smoking status correlates with ORR and indeed it was $22.5 \%$ for former/current smokers and $10.3 \%$ for never smokers. The median duration of response was 12.5 months for all patients, from 10.4 months for pretreated patients to 23.3 months for treatment-naïve patients. The median duration of progression free survival (PFS) was 3 months for pretreated patients and 6 months in treatment-naïve patients with 3.7 months across overall patients and the median duration of OS was 9.3 months for pretreated patients and not yet reached for untreated patients. In this trial treatmentrelated adverse events (AEs) occurred in $70.9 \%$ of patients $(n=351)$. Fatigue, decreased appetite and pruritus were the most common AEs and occurred in $10-20 \%$ of all patients. No clear difference was recorded between the incidence of AEs and the dose or schedule of pembrolizumab. AEs of grade 3 or higher were reported in $9.5 \%$ of patients $(n=47)$. Immunerelated AEs occurred in more than $2 \%$ of patients including hypothyroidism ( $\mathrm{n}=34$ patients, 6.9\%), hyperthyroidism $(\mathrm{n}=9$ patients, $1.8 \%)$, pneumonitis $(\mathrm{n}=18$ patients, $3.6 \%)$ and infusion-related reaction ( $n=10$ patients, $2 \%$ ). All the cases of hypothyroidism were treated successfully with medical therapy, a single case of infusion related reaction led to discontinuation of treatment. Only one case $(0.2 \%)$ of grade 5 lung disease was recorded. In this trial 824 of all screened patients $[1,143]$ were evaluable for PDL-1 expression. The prevalence of PDL-1 expression of $50 \%$ or higher was $23.2 \%$ in all patients (24.9\% in untreated patients and $22.7 \%$ in previously treated patients). According to PDL-1 expression the median PFS was 6.3 months for all patients with a proportion score of at least 50\% (6.1 months for previously treated patients and 12.5 months for untreated patients), the median OS was not yet reached. The median duration of response according to PDL-1 expression was similar to median PFS. Relevant difference in terms of ORR, PFS and OS has been recorded between patients with a proportion score of at least $50 \%$ and patients with a proportion score of $1-49 \%$ or $<1 \%$. Indeed the ORR was $42.5 \%$, PFS of 6.3 months and not reached OS for patients with proportion score of at least $50 \%$, whereas in patients with a proportion score of $1-49 \%$ the ORR was $16.5 \%$ with PFS of 4.1 months and OS of 10.6 months, similar data has been recorded also in patients with a proportion score $<1 \%$, with ORR of $10.7 \%$, PFS of 4.0 months and OS 10.4 months. 


\section{KEYNOTE-010 (44)}

From August 2013 to April 2015 a total of 1,034 patients which have progressed after two or more cycles of platinum-doublet chemotherapy, as well as an appropriate TKI for those with an EGFR-activating mutation or ALK gene rearrangement were enrolled in this phase $2 / 3$ trial. All the patients with an expression of PD-L1 on at least $1 \%$ of tumor cells were randomized to receive every 3 weeks pembrolizumab at the dose of 2 or $10 \mathrm{mg} / \mathrm{kg}$ or docetaxel at the dose of $75 \mathrm{mg} / \mathrm{m}^{2}$. In this trial was predefined a subgroup analysis for those patients defined strongly PDL1 positive (442 of 1,034) with an expression of PD-L1 on $\geq 50 \%$ of their tumor cells. The primary endpoints were OS and PFS. Safety, response rate and duration of response were the secondary endpoints. In the total population the median OS was 10.4 months [hazard ratio (HR) 0.71] for the pembrolizumab $2 \mathrm{mg} / \mathrm{kg}$ group, 12.7 months (HR 0.61) for pembrolizumab $10 \mathrm{mg} / \mathrm{kg}$ group versus 8.5 months for docetaxel group. In the subgroup defined PD-L1 strongly positive (with an expression of PD-L1 $\geq 50 \%$ ) the median OS for each arm was 14.9 month for $2 \mathrm{mg} / \mathrm{kg}$ arm, 17.3 months for $10 \mathrm{mg} / \mathrm{kg}$ arm and 8.2 months for docetaxel arm, with an HR of 0.54 and 0.50 for pembrolizumab 2 and $10 \mathrm{mg} / \mathrm{kg}$ arm versus docetaxel arm. PFS was significantly longer with pembrolizumab than with docetaxel in patients with tumor expression of PD-L1 $\geq 50 \%, 5.0$ months (HR 0.59) for $2 \mathrm{mg} / \mathrm{kg}$ arm and 5.2 months (HR 0.59) for $10 \mathrm{mg} / \mathrm{kg}$ arm versus 4.1 months for docetaxel arm. Also for patients with a PD-L1 expression until 50\% there was an improvement in term of PFS but not statistically significant. In the subgroup analysis, although with the limitations of this kind of analysis, benefit of pembrolizumab among the patients with EGFR-mutant status in terms of OS and PFS was not as confirmed as in other subgroups (OS: $\mathrm{HR}=0.88$; $95 \%$ CI, 0.45-1.70; PFS: HR =1.79; 95\% CI, 0.94-3.42). Similar data was also recorded for patients never smokers respect the former or current smokers in which pembrolizumab led to an improvement in OS and PFS.

\section{Ongoing trials}

Based on the results described above the pembrolizumab monotherapy compared to platinum-based chemotherapy is being evaluated as NSCLC first-line treatment in two clinical trials: KEYNOTE-024 (ClinicalTrials.gov NCT02142738) and KEYNOTE-042 (ClinicalTrials.gov NCTC02220894). Chemotherapy combinations with pembrolizumab are being explored in a phase 1/2 trial: KEYNOTE-021 (ClinicalTrials. gov NCT02039674). Pembrolizumab is being evaluated as monotherapy in a phase 3 trial in patients with non-metastatic NSCLC: KEYNOTE-091 (PEARLS) (ClincialTrial.gov NCT02504372). In the Table 1 are summarized ongoing trials.

\section{KEYNOTE-024}

Is an ongoing, randomized, open label, phase 3 study to assess efficacy and safety of pembrolizumab compared to standard of care (SOC) platinum-based chemotherapies in the treatment of patients with chemo-naïve, metastatic and strong expressing PD-L1 NSCLC. The primary hypothesis of this study is that patients with PD-L1 strong NSCLC will have a longer PFS, as assessed by Response Evaluation Criteria in Solid Tumors version 1.1 (RECIST 1.1) when treated with pembrolizumab than when treated with platinum-based chemotherapies. In this trial the pembrolizumab at the dose of $200 \mathrm{mg}$ every 3 weeks (arm A) for up 35 cycles or until documented progressive disease (PD) is compared to carboplatin plus paclitaxel every 3 weeks (arm B) or carboplatin plus pemetrexed every 3 weeks (arm C) or cisplatin plus pemetrexed every 3 weeks (arm D) or carboplatin every 3 weeks plus gemcitabine at the day 1 and 8 every 3 weeks (arm E) or cisplatin every 3 weeks plus gemcitabine at the day 1 and 8 every 3 weeks (arm F), according to histology. Patients with non-squamous histologies enrolled in arm B, C or D after 4-6 cycles may receive optional pemetrexed every 3 weeks as maintenance therapy until PD. For all the SOC arms is permitted the crossover to pembrolizumab treatment at the time of documented PD. This study started in October 2014.

\section{KEYNOTE-042}

In this ongoing, open label phase 3 study, patients with untreated, metastatic and PD-L1-positive NSCLC will be randomized to receive single agent pembrolizumab $200 \mathrm{mg}$ every 3 weeks for up to 35 cycles or platinum doublet chemotherapy (investigators can choice between carboplatin plus paclitaxel and carboplatin plus pemetrexed) for 4 to 6 cycles every 3 weeks, according to histology. Patients on the platinum-based chemotherapy arms with non-squamous tumor histologies after 4-6 cycles may receive pemetrexed as maintenance therapy. In this trial is permitted the crossover to pembrolizumab treatment for all the patients enrolled in platinum-based chemotherapy arms at the time of documented PD. The primary study hypothesis is that pembrolizumab prolongs OS compared to SOC chemotherapy for PD-L1 positive patients. The enrollment began in November 2014. 
Table 1 Ongoing clinical trial with pembrolizumab

\begin{tabular}{|c|c|c|c|c|c|}
\hline Name of study & Description & Phase & $\begin{array}{l}\text { Estimated } \\
\text { patients }\end{array}$ & $\begin{array}{l}\text { Line of } \\
\text { treatment }\end{array}$ & Regimen \\
\hline $\begin{array}{l}\text { KEYNOTE-024 } \\
\text { (NCT02142738) }\end{array}$ & $\begin{array}{l}\text { Study of pembrolizumab } \\
\text { (MK-3475) compared to platinum- } \\
\text { based chemotherapies in } \\
\text { participants with metastatic NSCLC }\end{array}$ & Phase 3 & $\begin{array}{l}\mathrm{N}=300 \\
\text { (PD-L1 strong } \\
\text { positive) }\end{array}$ & $1^{\text {st }}$ line & $\begin{array}{l}\text { Pembrolizumab } 200 \text { mg Q3W; } \\
\text { Carbo/Tax; Carbo/Pem or Cis/Pem; } \\
\text { Carbo/Gem or Cis/Gem }\end{array}$ \\
\hline $\begin{array}{l}\text { KEYNOTE-042 } \\
\text { (NCT02220894) }\end{array}$ & $\begin{array}{l}\text { Study of MK-3475 (pembrolizumab) } \\
\text { vs. platinum-based chemotherapy } \\
\text { for participants with PD-L1-positive } \\
\text { advanced or metastatic NSCLC }\end{array}$ & Phase 3 & $\begin{array}{l}\mathrm{N}=1,240 \\
\text { (PD-L1 positive) }\end{array}$ & $1^{\text {st }}$ line & $\begin{array}{l}\text { Pembrolizumab } 200 \text { mg Q3W; } \\
\text { Carbo/Tax; Carbo/Pem }\end{array}$ \\
\hline $\begin{array}{l}\text { KEYNOTE-021 } \\
\text { (NCT02039674) }\end{array}$ & $\begin{array}{l}\text { A study of pembrolizumab } \\
\text { (MK-3475) in combination with } \\
\text { chemotherapy or immunotherapy in } \\
\text { participants with lung cancer }\end{array}$ & $\begin{array}{l}\text { Phase } \\
1 / 2\end{array}$ & $\mathrm{~N}=308$ & $1^{\text {st }}$ line & $\begin{array}{l}\text { Pembrolizumab + Carbo/Tax; } \\
\text { pembrolizumab + Carbo/Tax + } \\
\text { bevacizumab; pembrolizumab } \\
\text { + carbo/Pem; pembrolizumab } \\
\text { + ipilimumab; pembrolizumab + } \\
\text { erlotinib; pembrolizumab + gefitinib }\end{array}$ \\
\hline $\begin{array}{l}\text { KEYNOTE-091 } \\
\text { (PEARLS) } \\
\text { (NCT02504372) }\end{array}$ & $\begin{array}{l}\text { Study of pembrolizumab } \\
\text { (MK-3475) vs. placebo for } \\
\text { participants with NSCLC after } \\
\text { resection with or without standard } \\
\text { adjuvant therapy }\end{array}$ & Phase 3 & $N=1,380$ & $\begin{array}{l}\text { Adjuvant } \\
\text { setting }\end{array}$ & $\begin{array}{l}\text { Pembrolizumab } 200 \text { mg Q3W for } \\
12 \text { months }\end{array}$ \\
\hline
\end{tabular}

Carbo/Tax, carbloplatin + paclitaxel; Carbo/Pem, carboplatin + pemetrexed; Carbo/Gem, carboplatin + gemcitabine; Cis/Gem, cisplatin + gemcitabine; Cis/Pem, cisplatin + pemetrexed; NSCLC, non-small cell lung cancer; PD-L1, programmed cell death-ligand 1.

\section{KEYNOTE-021}

The purpose of this phase $1 / 2$ study is to determine safety, tolerability, and efficacy of pembrolizumab in combination with chemotherapy or immunotherapy in participants with unresectable or metastatic NSCLC; preliminary data presented at American Society of Clinical Oncology 2015 (45) annual meeting have shown a manageable toxicity profile and an ORR about $30 \%$ and about $58 \%$ for the combination cohort pembrolizumab plus carboplatin/paclitaxel and pembrolizumab plus carboplatin/pemetrexed respectively. These data suggest that pembrolizumab + platinum-based chemotherapy has a reasonable safety profile and provides antitumor activity as front-line therapy for stage IIIB/IV NSCLC. Based on the promising ORR observed for pembrolizumab plus carboplatin/pemetrexed, this combination is being evaluated in a larger cohort. For the combination-cohort pembrolizumab plus ipilimumab, a fully human IgG1 monoclonal antibody against CTLA-4 approved for treatment of metastatic melanoma, the preliminary data also presented at the ASCO 2015 have shown an ORR of $55 \%$ with a disease control rate (complete response plus partial response plus stable disease $\geq 6$ weeks) of $100 \%$. The dose reduction from 10 to $2 \mathrm{mg} / \mathrm{kg}$ for pembrolizumab and from 3 to $1 \mathrm{mg} / \mathrm{kg}$ for ipilimumab due the data from nivolumab and ipilimumab did not appear to detrimentally interfere on efficacy. Also the safety profile of this combination cohort was acceptable with $66 \%$ of patients which experienced AEs but none able to lead to death or discontinuation.

\section{KEYNOTE-091 (PEARLS)}

In this phase 3 trial, patients with Stage IB/II-IIIA NSCLC who have undergone surgical resection (lobectomy or pneumonectomy) with or without adjuvant chemotherapy will be treated with pembrolizumab or placebo. The primary study hypothesis is that pembrolizumab will provide improved disease-free survival (DFS) versus placebo. The enrollment began in August 2015.

\section{Safety}

The Table 2 summarize the AEs occurred in KEYNOTE-001 and KEYNOTE-010. PD-1 immune checkpoint inhibitors are associated with immune-mediated adverse events (IrAEs) 
Table 2 AEs of KEYNOTE-001 and KEYNOTE-010

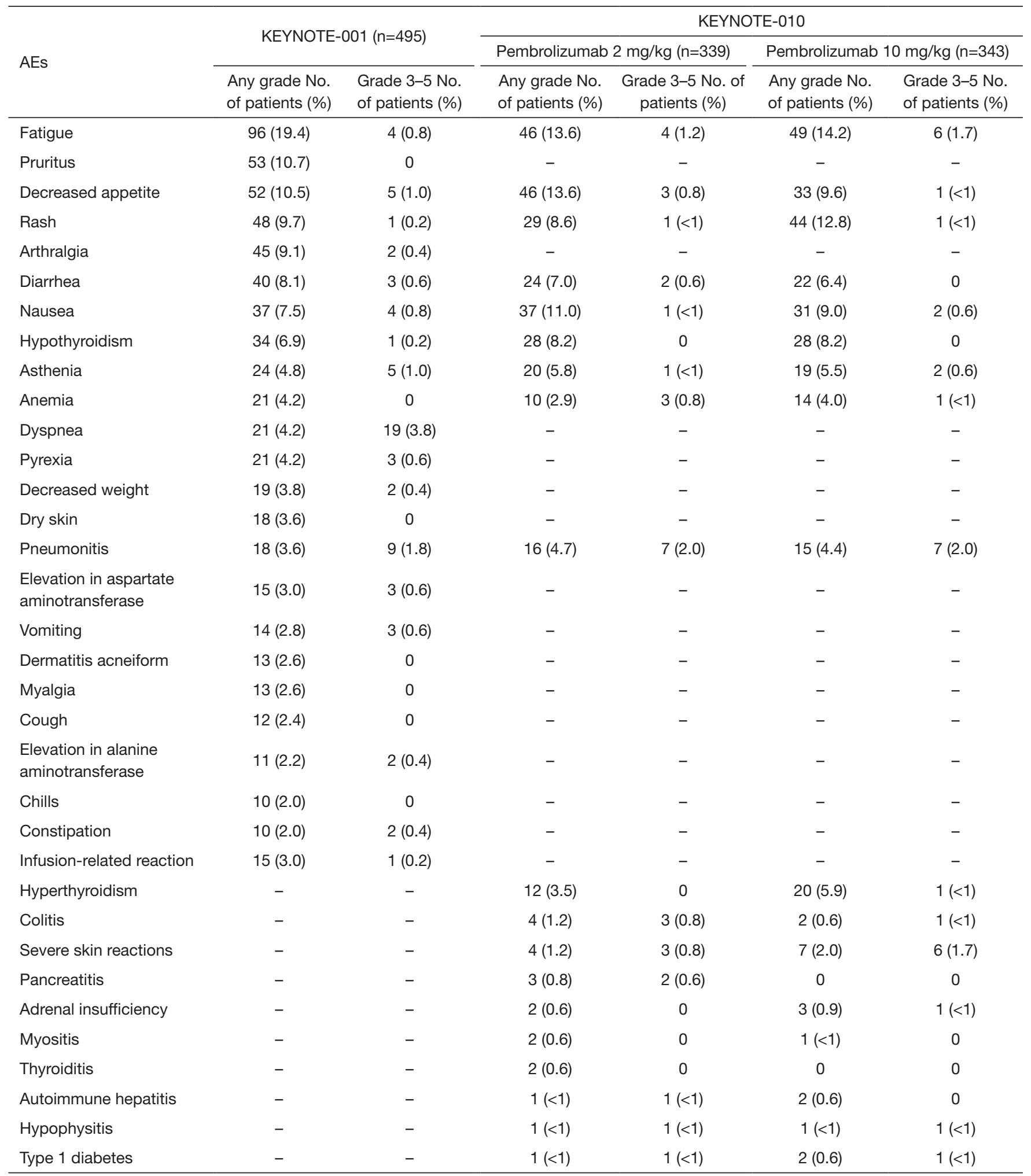

AEs, adverse event. 
$(42,46,47)$. Similar AEs were reported with ipilimumab (48) although the safety profile reported with PD-1 immune checkpoints inhibitors seems different. The mechanisms of action of the PD-1 immune checkpoint inhibitors may facilitate activation of potentially autoreactive T-cells, leading to inflammatory AEs across a range of tissue, indeed patients with a history of autoimmune disease or systemic immune suppression were excluded from clinical trials with PD-1 pathway inhibitors (49). Pembrolizumab is well tolerated and seems to have fewer gastrointestinal immune-mediated AEs of any grade: diarrhea $1-17 \%$ and colitis $1-4 \%(50,51)$, indeed most of individual grade 3-5 AEs have occurred in less than $10 \%$ of patients. From KEYNOTE-001 trial fatigue, pruritus, decreased appetite, rash, arthralgia, diarrhea nausea and hypothyroidism were the most detectable AEs of any grade. Most relevant were the dose-limiting immune-related AEs, although the frequency is low these can result in remarkable morbidity. IrAEs can happen at any time during the treatment and there is no dependence with dosage, these kinds of AEs include autoimmune hypophysitis, thyroid disorders and type 1 diabetes mellitus. Have been also described pneumonitis that may worsen lung function of patients who already have a respiratory impairment. Other IrAEs reported during treatment with pembrolizumab were colitis, hepatitis and nephritis. Rare cases of severe dermatitis (frequency in $<1 \%$ of patients) have been reported including bullous pemphigoid, rhabdomyolysis, myasthenia-like syndrome and optic neuritis (43).

\section{Conclusions}

Pembrolizumab is an effective and safe treatment for patients affected by NSCLC, the cornerstone of its use is the KEYNOTE-001 trial. In this study 495 patients with advanced NSCLC have been treated, the ORR has been $19.4 \%$ and a median duration of response 12.5 months. These results become even more interesting when the PDL1 expression in at least $50 \%$ of tumor cells is select as the cut-off, in this case the ORR reaches $45.2 \%$ with a median PFS of 6.3 months. Furthermore, both in previously treated patients and untreated patients the ORR has been found similar when the expression of PD-L1 was in at least $50 \%$ of tumor cells. The data from KEYNOTE-001 study demonstrate that the high expression of PD-L1 correlates with better response rate and clinical outcome after treatment with pembrolizumab (43).

From KEYNOTE-010 trial, responses to pembrolizumab treatment have not been shown in all patients with an expression of PD-L1 at least 50\% suggesting the existence of other immunosuppressive mechanisms in tumor microenvironment (44). The immune response is inhibited by CTLA-4 and PD-1 pathways through complementary mechanisms; CTLA-4 checkpoint plays a role in the dampening initial T-cells activation, while PD-1 inhibits T-cells response within tissues (29). Synergistic antitumor activity of dual blockade of both CTLA-4 and PD-1 has been demonstrated in preclinical trial. The combination of nivolumab and ipilimumab in patients with advanced melanoma has already demonstrated significantly longer PFS compared to ipilimumab in monotherapy $(51,52)$, albeit the side effects recorded in the combination arm have been greater than ipilimumab alone. Therefore, clinical trials are underway to assess the possibility of creating association strategies with immunotherapy in combination with other immunotherapeutic agents, chemotherapy or molecular targeted agents.

\section{Acknowledgements}

None.

\section{Footnote}

Conflicts of Interest: The authors have no conflicts of interest to declare.

\section{References}

1. Herbst RS, Heymach JV, Lippman SM. Lung cancer. N Engl J Med 2008;359:1367-80.

2. Santabarbara G, Maione P, Rossi A, et al. Pharmacotherapeutic options for treating adverse effects of Cisplatin chemotherapy. Expert Opin Pharmacother 2016;17:561-70.

3. Rosell R, Carcereny E, Gervais R, et al. Erlotinib versus standard chemotherapy as first-line treatment for European patients with advanced EGFR mutation-positive non-small-cell lung cancer (EURTAC): a multicentre, open-label, randomised phase 3 trial. Lancet Oncol 2012;13:239-46.

4. Mok TS, Wu YL, Thongprasert S, et al. Gefitinib or carboplatin-paclitaxel in pulmonary adenocarcinoma. $\mathrm{N}$ Engl J Med 2009;361:947-57.

5. Sequist LV, Yang JC, Yamamoto N, et al. Phase III study of afatinib or cisplatin plus pemetrexed in patients with 
metastatic lung adenocarcinoma with EGFR mutations. J Clin Oncol 2013;31:3327-34.

6. Shaw AT, Kim DW, Nakagawa K, et al. Crizotinib versus chemotherapy in advanced ALK-positive lung cancer. N Engl J Med 2013;368:2385-94.

7. Kobayashi S, Boggon TJ, Dayaram T, et al. EGFR mutation and resistance of non-small-cell lung cancer to gefitinib. N Engl J Med 2005;352:786-92.

8. Swann JB, Smyth MJ. Immune surveillance of tumors. J Clin Invest 2007;117:1137-46.

9. Zitvogel L, Tesniere A, Kroemer G. Cancer despite immunosurveillance: immunoselection and immunosubversion. Nat Rev Immunol 2006;6:715-27.

10. Hanson HL, Donermeyer DL, Ikeda H, et al. Eradication of established tumors by CD8+ $\mathrm{T}$ cell adoptive immunotherapy. Immunity 2000;13:265-76.

11. Klebanoff CA, Gattinoni L, Torabi-Parizi P, et al. Central memory self/tumor-reactive CD8+ T cells confer superior antitumor immunity compared with effector memory $\mathrm{T}$ cells. Proc Natl Acad Sci U S A 2005;102:9571-6.

12. Shimizu J, Yamazaki S, Sakaguchi S. Induction of tumor immunity by removing CD25+CD4+ T cells: a common basis between tumor immunity and autoimmunity. J Immunol 1999;163:5211-8.

13. Kerkar SP, Restifo NP. Cellular constituents of immune escape within the tumor microenvironment. Cancer Res 2012;72:3125-30.

14. Pardoll DM. The blockade of immune checkpoints in cancer immunotherapy. Nat Rev Cancer 2012;12:252-64.

15. Platonova S, Cherfils-Vicini J, Damotte D, et al. Profound coordinated alterations of intratumoral NK cell phenotype and function in lung carcinoma. Cancer Res 2011;71:5412-22.

16. Rabinovich GA, Gabrilovich D, Sotomayor EM. Immunosuppressive strategies that are mediated by tumor cells. Annu Rev Immunol 2007;25:267-96.

17. Sgambato A, Casaluce F, Sacco PC, et al. Anti PD-1 and PDL-1 Immunotherapy in the Treatment of Advanced Non- Small Cell Lung Cancer (NSCLC): A Review on Toxicity Profile and its Management. Curr Drug Saf 2016;11:62-8.

18. Fife BT, Bluestone JA. Control of peripheral T-cell tolerance and autoimmunity via the CTLA-4 and PD-1 pathways. Immunol Rev 2008;224:166-82.

19. Okazaki T, Chikuma S, Iwai Y, et al. A rheostat for immune responses: the unique properties of PD-1 and their advantages for clinical application. Nat Immunol 2013;14:1212-8.
20. Parry RV, Chemnitz JM, Frauwirth KA, et al. CTLA-4 and $\mathrm{PD}-1$ receptors inhibit $\mathrm{T}$-cell activation by distinct mechanisms. Mol Cell Biol 2005;25:9543-53.

21. Riley JL, Mao M, Kobayashi S, et al. Modulation of TCRinduced transcriptional profiles by ligation of CD28, ICOS, and CTLA-4 receptors. Proc Natl Acad Sci U S A 2002;99:11790-5.

22. Rudd CE, Taylor A, Schneider H. CD28 and CTLA-4 coreceptor expression and signal transduction. Immunol Rev 2009;229:12-26.

23. Lee HE, Chae SW, Lee YJ, et al. Prognostic implications of type and density of tumour-infiltrating lymphocytes in gastric cancer. Br J Cancer 2008;99:1704-11.

24. Liotta F, Gacci M, Frosali F, et al. Frequency of regulatory $T$ cells in peripheral blood and in tumour-infiltrating lymphocytes correlates with poor prognosis in renal cell carcinoma. BJU Int 2011;107:1500-6.

25. Suzuki H, Chikazawa N, Tasaka T, et al. Intratumoral CD8(+) T/FOXP3 (+) cell ratio is a predictive marker for survival in patients with colorectal cancer. Cancer Immunol Immunother 2010;59:653-61.

26. Chew V, Tow C, Teo M, et al. Inflammatory tumour microenvironment is associated with superior survival in hepatocellular carcinoma patients. J Hepatol 2010;52:370-9.

27. P9. her M, Braun M, Friedrichs N, et al. Foxp3(+) cell infiltration and granzyme $\mathrm{B}(+) / F o x p 3(+)$ cell ratio are associated with outcome in neoadjuvant chemotherapytreated ovarian carcinoma. Cancer Immunol Immunother 2010;59:909-19.

28. Park JJ, Omiya R, Matsumura Y, et al. B7-H1/CD80 interaction is required for the induction and maintenance of peripheral T-cell tolerance. Blood 2010;116:1291-8.

29. Rozali EN, Hato SV, Robinson BW, et al. Programmed death ligand 2 in cancer-induced immune suppression. Clin Dev Immunol 2012;2012:656340.

30. Zou W, Chen L. Inhibitory B7-family molecules in the tumour microenvironment. Nat Rev Immunol 2008;8:467-77.

31. Dong H, Zhu G, Tamada K, et al. B7-H1, a third member of the B7 family, co-stimulates T-cell proliferation and interleukin-10 secretion. Nat Med 1999;5:1365-9.

32. Brahmer JR, Drake CG, Wollner I, et al. Phase I study of single-agent anti-programmed death-1 (MDX-

1106) in refractory solid tumors: safety, clinical activity, pharmacodynamics, and immunologic correlates. J Clin Oncol 2010;28:3167-75.

33. Iwai $Y$, Ishida $M$, Tanaka $Y$, et al. Involvement of PD-L1 on tumor cells in the escape from host immune system 
and tumor immunotherapy by PD-L1 blockade. Proc Natl Acad Sci U S A 2002;99:12293-7.

34. Topalian SL, Hodi FS, Brahmer JR, et al. Safety, activity, and immune correlates of anti-PD-1 antibody in cancer. $\mathrm{N}$ Engl J Med 2012;366:2443-54.

35. Rizvi NA, Hellmann MD, Snyder A, et al. Cancer immunology. Mutational landscape determines sensitivity to PD-1 blockade in non-small cell lung cancer. Science 2015;348:124-8.

36. Cancer Genome Atlas Research Network. Comprehensive genomic characterization of squamous cell lung cancers. Nature 2012;489:519-25.

37. Gettinger SN, Horn L, Gandhi L, et al. Overall Survival and Long-Term Safety of Nivolumab (Anti-Programmed Death 1 Antibody, BMS-936558, ONO-4538) in Patients With Previously Treated Advanced Non-Small-Cell Lung Cancer. J Clin Oncol 2015;33:2004-12.

38. Brahmer J, Reckamp KL, Baas P, et al. Nivolumab versus Docetaxel in Advanced Squamous-Cell Non-Small-Cell Lung Cancer. N Engl J Med 2015;373:123-35.

39. Borghaei H, Paz-Ares L, Horn L, et al. Nivolumab versus Docetaxel in Advanced Nonsquamous Non-Small-Cell Lung Cancer. N Engl J Med 2015;373:1627-39.

40. Brahmer JR, Tykodi SS, Chow LQ, et al. Safety and activity of anti-PD-L1 antibody in patients with advanced cancer. N Engl J Med 2012;366:2455-65.

41. Patnaik A, Kang SP, Rasco D, et al. Phase I Study of Pembrolizumab (MK-3475; Anti-PD-1 Monoclonal Antibody) in Patients with Advanced Solid Tumors. Clin Cancer Res 2015;21:4286-93.

42. Robert C, Ribas A, Wolchok JD, et al. Anti-programmeddeath-receptor-1 treatment with pembrolizumab in ipilimumab-refractory advanced melanoma: a randomised dose-comparison cohort of a phase 1 trial. Lancet 2014;384:1109-17.

43. Garon EB, Rizvi NA, Hui R, et al. Pembrolizumab for the

Cite this article as: Santabarbara G, Maione P, Rossi A, Palazzolo G, Gridelli C. The role of pembrolizumab in the treatment of advanced non-small cell lung cancer. Ann Transl Med 2016;4(11):215. doi: 10.21037/atm.2016.05.64 treatment of non-small-cell lung cancer. N Engl J Med 2015;372:2018-28.

44. Herbst RS, Baas P, Kim DW, et al. Pembrolizumab versus docetaxel for previously treated, PD-L1-positive, advanced non-small-cell lung cancer (KEYNOTE-010): a randomised controlled trial. Lancet 2016;387:1540-50.

45. Papadimitrakopoulou V, Patnaik A, Borghaei H, et al. Pembrolizumab (pembro; MK-3475) plus platinum doublet chemotherapy (PDC) as front-line therapy for advanced non-small cell lung cancer (NSCLC): KEYNOTE-021 Cohorts A and C. J Clin Oncol 2015;33:abstr 8031.

46. Topalian SL, Sznol M, McDermott DF, et al. Survival, durable tumor remission, and long-term safety in patients with advanced melanoma receiving nivolumab. J Clin Oncol 2014;32:1020-30.

47. Robert C, Long GV, Brady B, et al. Nivolumab in previously untreated melanoma without BRAF mutation. N Engl J Med 2015;372:320-30.

48. Hodi FS, O'Day SJ, McDermott DF, et al. Improved survival with ipilimumab in patients with metastatic melanoma. N Engl J Med 2010;363:711-23.

49. Medina PJ, Adams VR. PD-1 Pathway Inhibitors: Immuno-Oncology Agents for Restoring Antitumor Immune Responses. Pharmacotherapy 2016;36:317-34.

50. Robert C, Schachter J, Long GV, et al. Pembrolizumab versus Ipilimumab in Advanced Melanoma. N Engl J Med 2015;372:2521-32.

51. Larkin J, Chiarion-Sileni V, Gonzalez R, et al. Combined Nivolumab and Ipilimumab or Monotherapy in Untreated Melanoma. N Engl J Med 2015;373:23-34.

52. Curran MA, Montalvo W, Yagita H, et al. PD-1 and CTLA-4 combination blockade expands infiltrating $\mathrm{T}$ cells and reduces regulatory $\mathrm{T}$ and myeloid cells within B16 melanoma tumors. Proc Natl Acad Sci U S A 2010;107:4275-80. 